\title{
Modulation of autophagy by miRNAs
}

\author{
Yunha Kim ${ }^{1}$, Junghee Lee ${ }^{2,3} \mathcal{E}$ Hoon Ryu ${ }^{2,3, *}$ \\ ${ }^{1}$ Center for Neuromedicine, Korea Institute of Science and Technology, Seoul 136-791, Korea, ${ }^{2}$ VA Boston Healthcare System, Boston, MA \\ 02130, USA, ${ }^{3}$ Boston University Alzheimer's Disease Center and Department of Neurology, Boston University School of Medicine, Boston, \\ MA 02118, USA
}

\begin{abstract}
MicroRNAs (miRNAs) can regulate the expression of genes that are involved in multiple cellular pathways. However, their targets and mechanism of action associated with the autophagy pathway are not fully investigated yet. EWSR1 (EWS RNA-Binding Protein 1/Ewing Sarcoma Break Point Region 1) gene encodes a RNA/DNA binding protein that is ubiquitously expressed and plays roles in numerous cellular processes. Recently, our group has shown that EWSR1 deficiency leads to developmental failure and accelerated senescence via processing of miRNAs, but its role in the regulation of autophagy remains elusive. In this context, we further investigated and found that EWSR1 deficiency triggers the activation of the DROSHA-mediated microprocessor complex and increases the levels of miR125a and miR351, which directly target Uvrag. Interestingly, the miR125a- and miR351-targeted reduction of Uvrag led to the inhibition of autophagy in both ewsr1 knockout (KO) MEFs and ewsr1 KO mice. In summary, our study demonstrates that EWSR1 is associated with the posttranscriptional regulation of $U v$ rag via miRNA processing. The regulation of autophagy pathway in miRNAs-Uvrag-dependent manner provides a novel mechanism of EWSR1 deficiency-related cellular dysfunction. [BMB Reports 2015; 48(7): 371-372]
\end{abstract}

*Corresponding author. E-mail: hoonryu@bu.edu

http://dx.doi.org/10.5483/BMBRep.2015.48.7.121

Received 20 June 2015

Keywords: Autophagy, EWSR1, miR125a, miR351, UVRAG

Abbreviations: ATG5, autophagy related 5; ATG12, autophagy related 12; ATG14, autophagy related 14; BECN1, beclin 1; EWS, Ewing's Sarcoma; EWSR1, EWS RNA-binding protein 1/ Ewing Sarcoma Break Point Region 1; Ewsr ${ }^{+/+}$, Ewsr1 wild type; ewsr $1^{-1}$, Ewsr1 homozygous knock out; LAMP, lysosomal-associated membrane protein; MAP1LC3/LC3, microtubule-associated protein 1 light chain 3; MEF, mouse embryonic fibroblast; miRNA, microRNA; pri-miRNA, primary transcript miRNA; RNA-seq, whole transcriptome sequencing; siRNA, small interfering RNA; UVRAG, UV radiation resistance associated

Perspective to: Yunha Kim et al (2015), Uvrag targeting by Mir125a and Mir351 modulates autophagy associated with Ewsr1 deficiency. Autophagy. 11(5):796-811.
MiRNAs are known to target autophagy-related genes and negatively regulate their activities. Moreover, miRNAs modulate autophagy at different stages such as autophagic induction, vesicle nucleation, vesicle elongation and completion, by targeting autophagy complexes via different miRNAs. Although a growing body of evidence indicates that miRNAs modulate autophagy, their target genes and precise roles in the autophagy pathways have not been fully defined yet. Considering the significance of autophagy in pathological conditions, including cellular senescence and neurodegeneration, a study on the regulation of autophagy by EWSR1, a multifunctional protein, may provide a better understanding of the autophagic signaling pathway. In this regard, we investigated how EWSR1 regulates the autophagy-related cellular processes, using whole transcriptome (mRNA) sequencing combined with miRNA arrays to analyze genes and miRNAs that are significantly altered in ewsr1 $1^{-1-}$ mouse embryonic fibroblasts (MEFs). We identified the transcriptome that are involved in the autophagy pathway and directly targeted by miRNAs. Notably, we discovered that miR351 and miR351 target Uvrag directly, and regulate autophagy. Our group further confirmed that decreased levels of UVRAG leads to autophagy inhibition.

Uvrag is a mammalian ortholog of yeast Vps38. It forms distinct complexes with BECN1 (mammalian ortholog of yeast Vps30/Atg6) and the class III phosphatidylinositol 3-kinase (whose catalytic subunit [PIK3C3] is the mammalian ortholog of yeast $\mathrm{Vps} 34$ ), contributing to both autophagosome formation and maturation. It is known that UVRAG deficiency decreases autophagy and raises uncontrolled cell proliferation. To date, it has not been established whether EWSR1 participates in the posttranscriptional regulation of UVRAG expression via DROSHA and miRNA-dependent pathways. Interestingly, we discovered that Uvrag mRNA is mainly regulated in the cytoplasm and is inversely correlated with elevated DROSHA levels in ewsr $1^{-1-}$ MEFs. Since we found that DROSHA is increased by EWSR1 deficiency, we hypothesized that UVRAG levels may be modulated by a DROSHA-miRNAependent pathway. We performed miRNA microarray analyses and identified that miR125a and miR351 are significantly increased in ewsr $1^{-1-}$ MEFs. Indeed, our group verified that Uvrag mRNA is a direct target of miR125a and miR351. Even though it has been proposed that Uvrag might be a potential target gene for miR351, no study has proven or validated that 


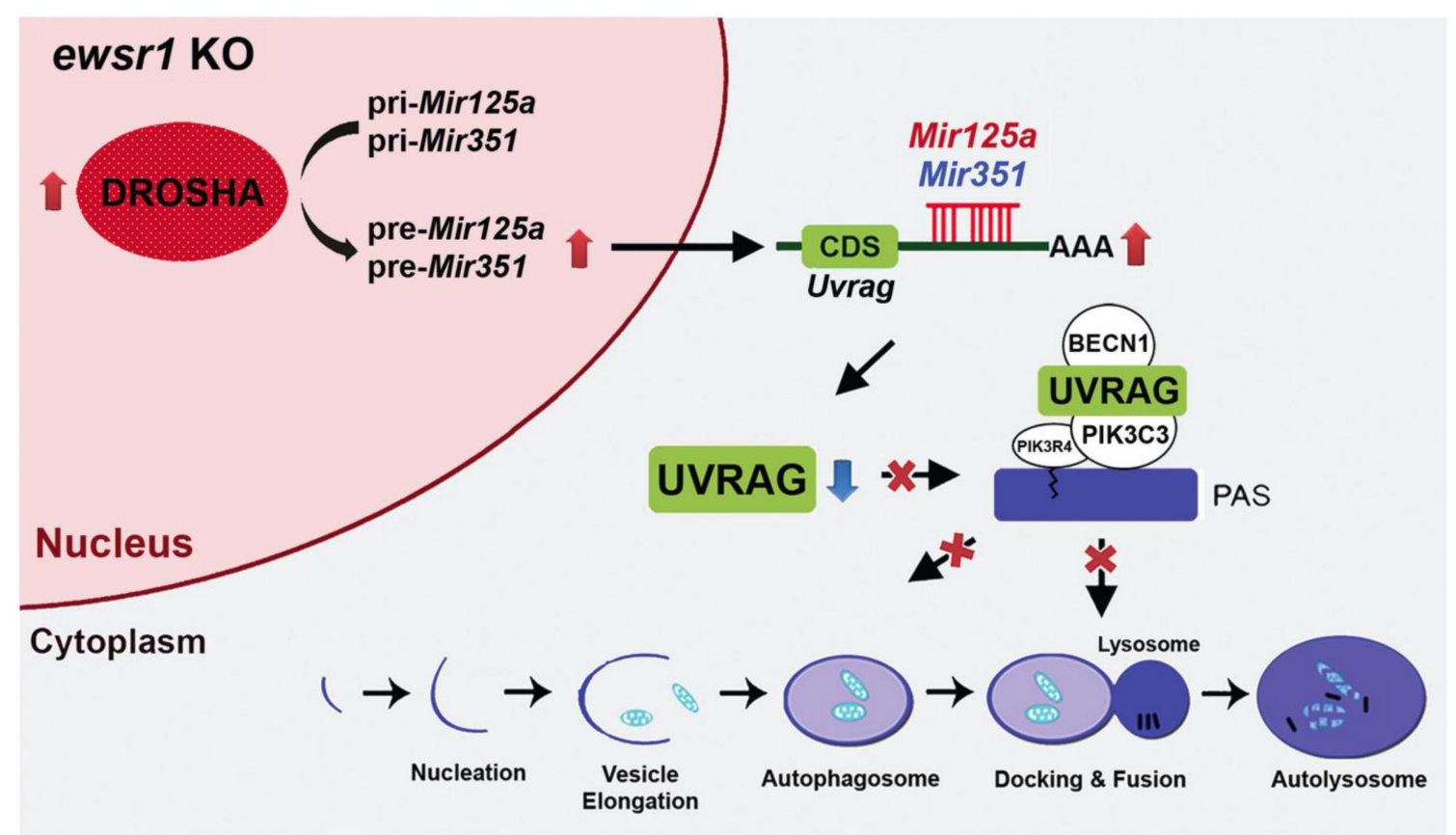

Fig. 1. A schema shows that autophagy is modulated through the posttranscriptional regulation of UVRAG by miRNAs under Ewsr1-deficient condition. Elevation of DROSHA level by EWSR1 deficiency triggers the processing of pri-miR125a and pri-miR351 to pre-miRNAs. Both miR125a and miR351 target and degrade Uvrag mRNA in the cytosol. Consequently, a reduction of UVRAG impairs UVRAG-dependent autophagy pathway. This figure is adopted from Autophagy (2015) 11 (5):796-811.

possibility. Our data show that Uvrag is a direct target of both miR125a and miR351, and that EWSR1 deficiency downregulates UVRAG via a miRNA-dependent pathway at the posttranscriptional level. Moreover, we confirmed that the levels of UVRAG and LC3-II (autophagy marker) are significantly reduced, while the levels of miR125a and miR351 are elevated in ewsr1 $\mathrm{KO}$ mice. This in vivo finding supports the in vitro data that EWSR1 deficiency leads to a reduction of autophagy (Fig. 1).

The assembly of core BECN1 and PIK3C3 initiates autophagy with BECN1-interacting proteins to form the autophagosome. The core complex exists in multiple forms with different BECN1-interacting proteins, UVRAG and ATG14, in a mutually exclusive manner. Although the direct role of UVRAG in autophagy is controversial, our current data clearly show that a reduction of UVRAG contributes to the deregulation of autophagy in the context of Ewsr1 deficiency, while ATG14, an alternative partner of UVRAG that binds to BECN1, is highly expressed in the absence of EWSR1. ATG14-containing complexes facilitate autophagosome nucleation and expansion, resulting in early stage autophagosome biosynthesis. Interestingly, we found that ATG14 and PIK3R4 were elevated, and also
LAMP1/2 (lysosomal-associated membrane protein 1/2) and four types of lysosomal enzymes (cathepsins) were increased in ewsr $1^{-1-}$ MEFs. Based on our data, we propose that increased LAMPs and cathepsins accelerate autolysosomal protein degradation, implying that the enhancing autophagic flux and increased ATG14 play a mechanistic role as BECN1 binding partners to form the core complex of autophagy, instead of UVRAG. However, it seems unlikely that increased ATG14 and the other molecules directly contribute to the deregulation of autophagy by Ewsr 1 deficiency and UVRAG reduction. In this paradigm, UVRAG may play a major regulatory role of autophagy under Ewsr1-deficiency conditions.

In conclusion, our results suggest that the reduced level of UVRAG by miRNAs deregulates autophagy and contributes to cellular dysfunction in developmental processes with Ewsr1 deficiency.

\section{ACKNOWLEDGEMENTS}

This study was supported by NIH R01 Grant (NS067283 to H.R.) and Brain Science Flagship Grant (2E25040 to H.R.) from Korea Institute of Science and Technology. 